\title{
Special quantitative methods for orienteering training in areas without orienteering map
}

\author{
HaoLing Zhang \\ School of Computer Science and Engineering \\ Chongqing University of Technology, Chongqing, China \\ hlzchn@gmail.com
}

Abstract: The objective of this study was to deal with the training problem of orienteering in the areas that suitable for orienteering but lack orienteering map since current training methods do not quantify the level of orienteering athletes. This study proposes to use the decomposition method to divide the problem of foot-orienteering into sub-parts and use the special quantitative methods to solve specific sub-problems. Experiments results show that the proficiency of the participants' sub-skills is increasing. All participants took part in the Orienteering Competition of the National Student, and the team performance increased by nearly $13 \%$ and the proportion of the normal athletes who gain the ranking was increased from $0 \%$ to $60 \%$. The results of this study demonstrate that applying the decomposition method and the above training methods are effective and reasonable, it also offers a useful solution for coaches to operate training and quantify easily. Keywords: deep thinking sport, decomposition method, Arithmetic Mean Running, Bleep Test in Plum-blossom, Bleep Test with Legend 


\section{Introduction}

Orienteering is a competitive and skilled sport who runs and navigates from the current target to the next target in the unfamiliar area by using orienteering map (Bengtsson \& Atkinson, 1977; Sension-Hall, 2011) and compass, as shown in Figure 1a, 1b. The orienteering map is based on corresponding high-precision militarized map and is drawn with given symbols (Zentai, 2014), as shown in Figure 1a, 1c. The orienteering map plays a vital role in this sport so that it's difficult to carry out professional training work without orienteering maps (Fairbairn, 1990).

Figure $1 \mathrm{~b}, 1 \mathrm{~d}$ give the illustration of the orienteering. We can see it requires athletes to make reasonable choices and adjustments for current motion state and the next route according to goals, environment, and circumstances (Creagh \& Reilly, 1997). In the basic technical training, using orienteering map is the most traditional and effective way of training (Cych, 2006; Tønnessen et al., 2015). In general, the competence of a trainee is positively related to the usage of different orienteering map. Moreover, the effect of training will be reduced with the increase using of the same orienteering map in the same area (Komiyama et al., 2015). Hence, preparing enough number of orienteering map is one of the most important tasks in actual orienteering training (Macquet, Eccles, \& Barraux, 2012).

Owing to the high complexity and other reasons of drawing and updating orienteering maps, many countries have studied how to make orienteering maps quickly and accurately. Modern mapping methods based on global navigation satellite system (Dodge, 2017), unmanned aerial vehicle (Chi, Lee, \& Tsai, 2016), unpowered exoskeleton (Zhang, Zhang, Huang, \& Liang, 2018), and other technology are becoming mature. Because drawing orienteering maps takes the high cost to learn, long time to draw, short intervals to update, the orienteering map is still increased slowly from most of the areas in the world (Maling, 2016). Therefore, it is a challenging and critical work 
for reasonable and effective orienteering training in areas which began later, lacked related orienteering infrastructure and orienteering maps.

In recent years, research on replacing orienteering maps for training is also under way. The two practical alternatives are by using satellite map and virtual game. In China, PaoXiang (www.ipaox.com) is a smartphone platform with satellite map, which is used for training orienteering athletes. However, sometimes the satellite map is old or incomplete (Krauß, 2015) and with the Global Navigation Satellite System error (Johnston, Watsford, Kelly, Pine, \& Spurrs, 2014) so that it is difficult to distinguish the level of orienteering ability between trainees under the influence of satellite map error. With the increasing of virtual technology, some virtual orienteering game has been developed (Wan, 2014). But the promotion is slow even limited.

The competitive level of orienteering is the comprehensive expression of many skills such as physical ability distribution, route selection, terrain analysis, and so on. But there is few research works on building systematic and quantitative training evaluation in orienteering training methods.

In this study, the whole of foot-orienteering skill is decomposed into a number of sub-problem. The training is carried by separate skills first and combined at the end. We propose the quantitative evaluation methods (Wan, 2014) and design the specific experiment. In addition, we develop the APP (Umek \& Kos, 2016) for our proposed scheme named 'Special Training in Orienteering' (STO). In training process, trainees can use STO to carry out the orienteering training, and the STO returns the quantitative data of the related subjects. Through analyzing the real-time training data and the national competition scores feedback from trainees, the training programs and quantitative methods of this study is reasonable and effective to evaluate and improve the orienteering skill of trainees. 


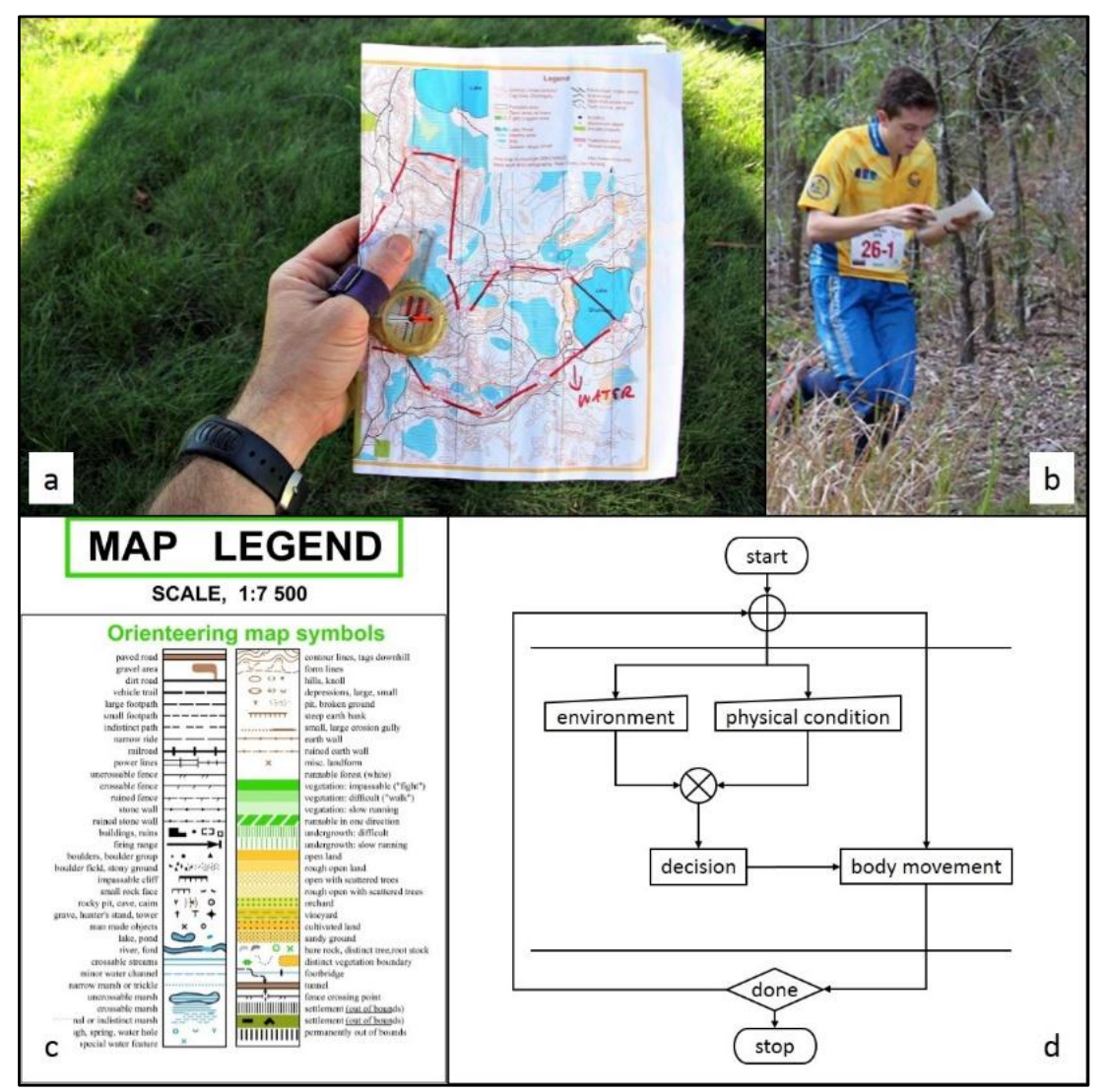

Figure 1. Illustration of the orienteering

\section{Method}

\section{Experimental Approach to the Problem}

In order to make orienteering training effectively and reasonable in areas which began later, lacked related infrastructure and maps, according to different training objective (Gilhooly, Wood, Kinnear, \& Green, 1988), the whole skill was divided into several sub-skills as shown in Figure 2, which is taken from the papers (Boes, Harung, Travis, \& Pensgaard, 2014; Gilhooly et al., 1988; Guzmán, Pablos, \& Pablos, 2008; Lauenstein, Wehrlin, \& Marti, 2013; McNeill, 2014; Millet, Divert, Banizette, \& Morin, 2010; Roelofs et al., 2015) and the first-person videos of competition. Because among all the orienteering training skills, 'thinking under hypoxia', 'directional recognition' and 'prescribed legends recognition' are hard to quantitative evaluate during real-time training without orienteering map. But these sub-skills are critical aspects that affect the training state of the athlete. 
In this paper, the quantitative schemes and specific simulation experiment were proposed for three special sub-problems, and an APP was developed for athletes and coaches using in actual training.

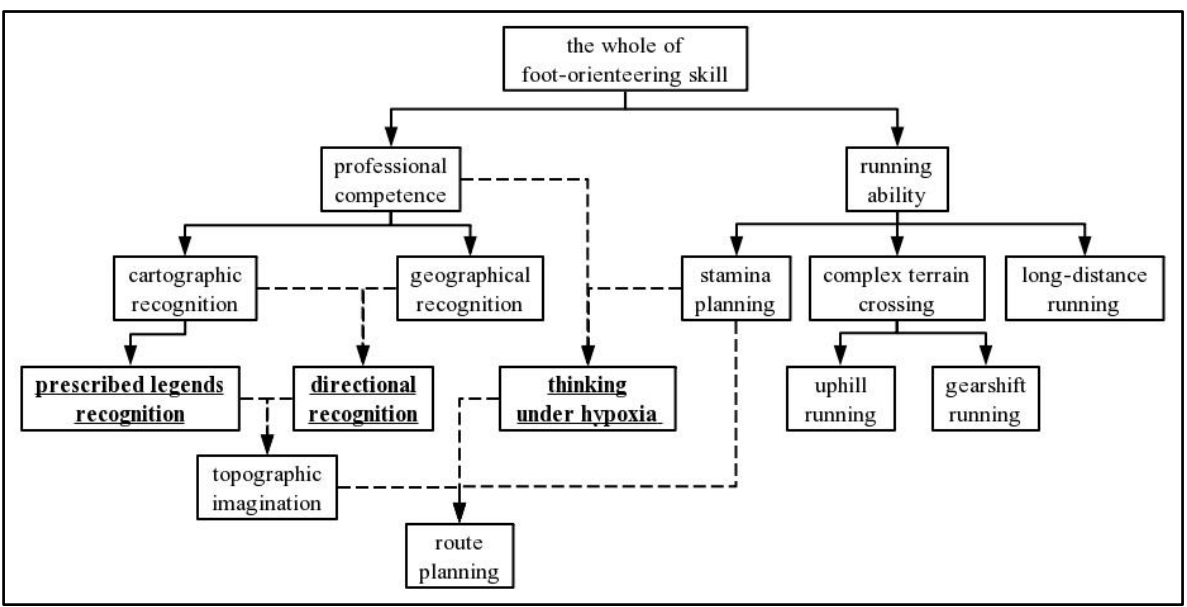

Figure 2. The dendrogram of foot-orienteering problem

(arrow of the lines refers to subclasses and dotted lines refer to the common influence of the superclass)

\section{Three Sub-skills in Foot-orienteering}

Considering that there are no specific experimental methods for testing. Therefore, this study made the special design of the above training problems.

\section{Method of Thinking under Hypoxia}

It is one of the core techniques to memorize all the features in the process of moving from the current target to the next in the state of under hypoxia (Asmaro, Mayall, \& Ferguson, 2013; Creagh \& Reilly, 1997). Status of the athletes can be described as Figure 1d.

Therefore, in view of the problem of hypoxic thinking, think about the orienteering map while moving was needed. Here, this study has designed the special original method which is named 'Arithmetic Mean Running' (AMR). The schematic diagram of AMR is shown in Figure 3(AMR), which describes the motion process and the APP interface of the AMR.

Because the persons who practice orienteering would make computation during their realtime training process, the computational objects of AMR cannot be too complex. In the design of AMR, this study found that the combination of the multiplication of single digit and the addition 
of ten digits could enable the trainee to memory and make calculations during the training and under hypoxia conditions. However, if there is too much calculation, it will cause the trainee to stop training because the excessive thinking (Asmaro et al., 2013). Therefore, it is important that thinking or selecting without running is not allowed.

\section{Method of Directional Recognition}

In current university sports teams, orienteering by using map as plum-blossom and multi-stage fitness test (Leger, Mercier, Gadoury, \& Lambert, 1988) are the improved training schemes. But this study believes that the simple use of map like plum-blossom is not sufficient to train the trainees' ability in the directional recognition. Because this method doesn't make trainees in the condition of hypoxia with high pressure (Asmaro et al., 2013), it cannot simulate the real game scene effectively. At the same time, the simple use of multi-stage fitness test cannot simulate the response ability (Zemková, Vilman, Kováciková, \& Hamar, 2013) in the real orienteering training.

Therefore, the method named 'Bleep Test in Plum-blossom' (BTP) was designed, which used to train the ability of directional recognition with response-ability. The schematic diagram of BTP shown in Figure 3(BTP), which describes the motion process and the APP interface of BTP.

There is a known fact that if one map is multi-used, the effect of training will decrease. Therefore, directional selection for the next target in BTP is implemented randomly. Moreover, the next line will be displayed in the current route after the end of time. The problem of the miscellaneous route is avoided.

\section{Method of Prescribed Legends Recognition}

In the orienteering sport, remembering orienteering symbols which is shown in Figure 1(c) is very important, because it is the premise of trainee to judge on route feasibility and plans the optimal 
path effectively. But currently, most of the training schemes do this after training, making trainee memory orienteering symbols in the static condition and it is not advisable.

Therefore, in order to train the real-time response of the subjects, this study proposes the quantitative method named 'Bleep Test with Legend' (BTL), according to the reappearance rule of orienteering map. The diagram of BTL is shown in Figure 3.BTL, which describes the motion process and the APP interface of the BTL. In the course of training, the appearance of a legend is also random. In this way, the different memory of all the examples is avoided.

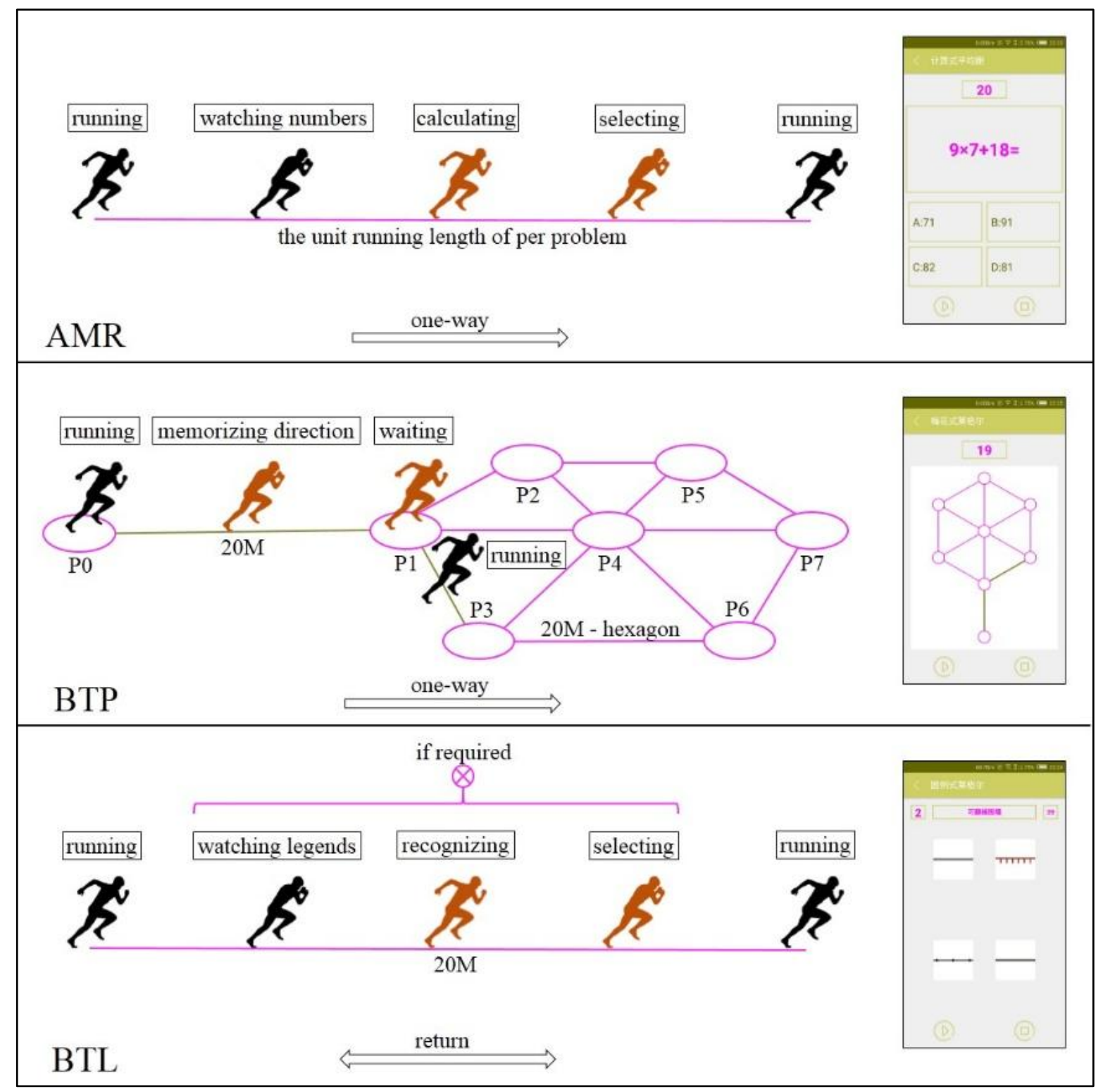

Figure 3. The schematic diagram of three proposed quantitative schemes for the three sub-skills 


\section{Design of the Three Quantitative Methods}

In the last part, this paper elaborated the design concept and the movement mode of the three training methods. In here, this paper will elaborate on how to design these methods by using the idea of engineering. And each method has 4 incremental levels in the STO, and the specific parameters will be displayed on the public web site, and this article does not describe it more. Moreover, it is worth noting that the turn time control of BTP and BTL is same as that of multistage fitness test. Therefore, the process named 'MSFT' in Figure 4 refers to it.

\section{Design of Arithmetic Mean Running}

According to the model in AMR, the calculation of the proposed as the formula (1):

$$
D_{r}=A \times B+C ; A, B \in[0,9] \& C \in[-99,99]
$$

$A, B$ and $C$ is the number for calculation which outputted randomly and obtains $D_{r}$ which is the right answer. It is shown in Figure 3(AMR). And the three error answers $D_{w 1}, D_{w 2}$ and $D_{w 3}$ was calculated as the formula (2):

$$
D_{w 1}=D_{r}+1 ; D_{w 2}=D_{r}+10 ; D_{w 3}=D_{r}-10
$$

$D_{r}, D_{w 1}, D_{w 2}$ and $D_{w 3}$ are arranged randomly in the interface and the correct answer option $R$ is saved so that the trainee is proofread by option position matching after clicking. Detail flowchart of AMR is shown in Figure 4.

This study has discussed the suitability of the solution for the wrong answer. Through simple tests, it is the same difficulty for getting the correct answer by direct calculation and getting the correct answer by using the results. So it is not to worry that the way of the trainee how to get the right answer. Moreover, when the trainee makes an option erroneously, the software will make a prompt and update the record of the errors number. After the situation, the total number of choose 
items will not be reduced, the trainee must complete an item as the punishment. This study thinks that this method of punishment refer to the path correction in the process of orienteering.

\section{Design of Bleep Test in Plum-blossom}

As shown in Figure 3(BTP), this study sets 8 positions as $P_{0} \sim P_{7}$, and $P_{0}$ is the terminal point. This study uses the breadth-first search (Cormen, Leiserson, Rivest, \& Stein, 2001) for the select visiting position randomly, and the path is not allowed to turn back. Based on the current visited position $P_{n}$, it can gets other visiting positions through the data structure in the sequential table, removing the last visited position $P_{c}$ and $P_{0}$, and gets the next visiting location $P_{f}$ by randomly. Detail flowchart of BTP is shown in Figure 4.

In order to make the training process more orderly, $P_{0}$ is designed as the last visit point. Therefore, the approach of this study is to limit the visiting position selection which starts from the third reciprocal visited position. The visited position of reciprocal fourth is divided into two categories, that is $P_{4}$ or is not $P_{4}$. If it is $P_{4}$, the next two paths will be created and one of them will be chosen randomly: $P_{2} \rightarrow P_{1} \rightarrow P_{0}$ and $P_{3} \rightarrow P_{1} \rightarrow P_{0}$. If it is not $P_{4}$, the next path will be created: $P_{4} \rightarrow P_{1} \rightarrow P_{0}$

\section{Design of Bleep Test with Legend}

After obtaining relevant examine and verify by orienteering experts in China, 84 types of legends are selected in BTL. These legends are all enlarged by using PHOTOSHOP so that trainees can judge them clearly and introduced in STO. It controls redundant error variables. Detail flowchart of BTL is shown in Figure 4.

The implementation of BTL is similar to AMR. The difference is that the calculation model of BTL uses the clear legends, and the single process also has the time control of multi-stage fitness test. The implementation scheme of the random selection is to select these legends randomly 
without repetition, which can be randomly assigned to 4 mapping object identifiers: $i_{1}, i_{2}, i_{3}$ and $i_{4}$. And these 4 identifiers will be selected as the right option $i_{r}$ randomly. After that, 4 randomly selected pictures $\mathrm{P}\left(i_{1}\right), \mathrm{P}\left(i_{2}\right), \mathrm{P}\left(i_{3}\right), \mathrm{P}\left(i_{4}\right)$ and the illustration $\mathrm{S}\left(i_{r}\right)$ are displayed in the STO by program mapping. The interface is shown in Figure 3(BTL).

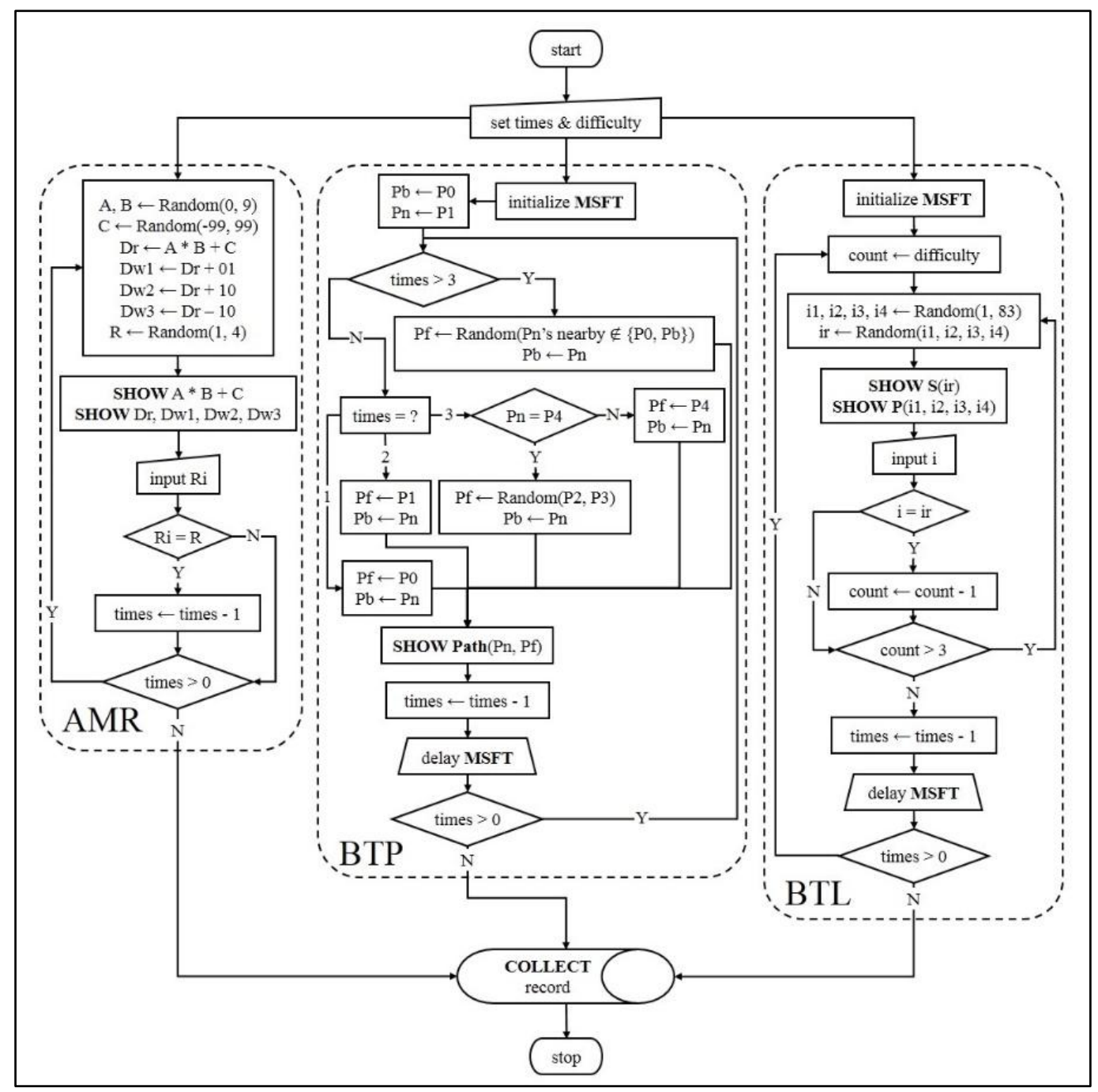

\section{Participants}

Figure 4. Flowchart of AMR, BTP, and BTL

There were 4 male and 4 female athletes participated in this study. Among them, 1 male and 2 female athletes who had participated in Orienteering Competition of the National Student. All participants were provided written informed consent and their ages are between 19 and 22 . This study was approved by Physical Education Department of Chongqing University of Technology. 


\section{Procedures}

From July 2, 2017, the preliminary introduction and detailed demonstration for STO was conducted, and the daily test and training plan are described in detail. The main training program is shown in Figure 5. Due to the high temperature, the training time was arranged at 06:00-08:00 per day. Before the main training program, the participants warm up at jog for $2000 \mathrm{~m}$ and stretch for 15 minutes.

The core of this study is to design and derivation of the three method, so there is no restriction on nutrition, hydration and consumed oxygen of the participants during the whole experiment. Such restrictions are expected to be carried out in subsequent experiments.

\begin{tabular}{|c|c|c|c|c|c|c|c|c|c|c|c|c|c|c|c|c|c|c|}
\hline \multirow{3}{*}{$\begin{array}{c}\text { AMR } \\
\text { BTP }\end{array}$} & \multirow{3}{*}{$\begin{array}{c}2017 / 7 / 4 \\
2000 \\
4 \times 25\end{array}$} & \multirow{2}{*}{\multicolumn{2}{|c|}{$\begin{array}{c}2017 / 7 / 5 \\
3000\end{array}$}} & \multirow{2}{*}{\multicolumn{2}{|c|}{$\frac{2017 / 7 / 6}{4000}$}} & \multicolumn{2}{|c|}{$2017 / 7 / 7$} & \multicolumn{2}{|c|}{$2017 / 7 / 8$} & \multicolumn{2}{|c|}{$2017 / 7 / 9$} & \multicolumn{2}{|c|}{$2017 / 7 / 10$} & \multirow{3}{*}{$\begin{array}{c}2017 / 7 / 11 \\
6000 \\
1 \times 25\end{array}$} & \multicolumn{2}{|c|}{$2017 / 7 / 12$} & \multirow{2}{*}{\multicolumn{2}{|c|}{$\begin{array}{c}2017 / 7 / 13 \\
4000\end{array}$}} \\
\hline & & & & & & \multirow{2}{*}{\begin{tabular}{c|}
3000 \\
$2 \times 45$
\end{tabular}} & \multirow{2}{*}{\begin{tabular}{|c|}
1000 \\
$2 \times 35$
\end{tabular}} & \multirow{2}{*}{$\frac{2000}{2 \times 45}$} & \multirow{2}{*}{$\begin{array}{r}2000 \\
2 \times 25\end{array}$} & \multirow{2}{*}{$\frac{1000}{2 \times 45}$} & \multirow{2}{*}{\begin{tabular}{|c|}
3000 \\
$1 \times 35$
\end{tabular}} & \multirow{2}{*}{$\begin{array}{r}1000 \\
1 \times 45\end{array}$} & \multirow{2}{*}{$\frac{4000}{1 \times 35}$} & & \multirow{2}{*}{\begin{tabular}{|l}
2000 \\
$1 \times 60$
\end{tabular}} & \multirow{2}{*}{$\begin{array}{r}3000 \\
1 \times 45\end{array}$} & & \\
\hline & & $3 \times 35$ & $2 \times 25$ & $3 \times 35$ & $2 \times 25$ & & & & & & & & & & & & $1 \times 45$ & $1 \times 35$ \\
\hline BTL & $2 \times 15$ & $2 \times 20$ & $2 \times 25$ & $2 \times 20$ & $2 \times 25$ & $2 \times 25$ & $2 \times 25$ & $2 \times 35$ & $2 \times 25$ & $3 \times 35$ & $2 \times 25$ & $1 \times 35$ & $2 \times 35$ & $1 \times 25$ & $1 \times 35$ & $2 \times 35$ & $1 \times 35$ & $1 \times 35$ \\
\hline \multicolumn{19}{|l|}{ Level 1} \\
\hline \multicolumn{19}{|l|}{ Level 2} \\
\hline \multicolumn{19}{|l|}{ Level 3} \\
\hline Level 4 & & & & & & & & & & & & & & & & & & \\
\hline
\end{tabular}

\section{Data Collected}

Figure 5. The training arrangement for orienteering team

All training records have saved in the file from each participant's smartphone. And each record can be uploaded to a computer. The record contains 5 parts: 'exact start time', 'subject type', 'difficulty', 'count of correct' and 'count of errors'. It is worth noting that if the exact start time between the two plans is too close, it may be the data falsification.

\section{Statistical Analyses}

The quantitative analysis about score correlation of samples is the ratio of completion, all data is normalized. In this study, since the interaction between the three methods is not considered, 
separate calculations are taken. Multiple records for the participant of the same subject per day are calculated by using average value. And the proficiency is normalized for each participant.

With the increase of exercise intensity and task difficulty, the error rate will increase continuously (Komiyama et al., 2015). Thus, the amount of sample used per single record is the product of the exercise intensity and the task difficulty. Formula of proficiency for single sample is shown in formula (3):

$$
P=\frac{C}{C+E} \times \frac{D}{4}
$$

Where $C$ is the correct execution number of a single subject, $E$ is the error execution number of a single subject, $D$ is the difficulty of a single subject, and $P$ is the proficiency of a single subject.

In addition, the formula of normalization is shown in formula (4):

$$
N_{c}=\frac{P_{c}}{P_{M A X}}
$$

Where $N_{c}$ is the proficiency after normalized, $P_{c}$ is proficiency of single subject, $P_{M A X}$ is max proficiency of single subject in the experiment.

The deviation between the actual situation of the participants' training and the design scheme should be known. Therefore, the formula of deviation is shown in (5):

$$
\eta_{c}=\left(1-\frac{N_{c}}{N_{s}}\right) \times 100 \%
$$

Where $\eta_{c}$ is the deviation from the proficiency of training arrangement standard after normalized $\left(N_{S}\right)$ and $N_{C}$.

\section{Result}

It is necessary to elaborate the reasonableness and effectiveness of the decomposition method and the three methods fully by using some specific contrast test. These tests and its results are as follow: 


\section{Self-Promotion Contrast Test}

For each participant, the proficiency of the sub-skills is increasing as Figure 6(a), and the probability of failure is fluctuated, as shown in Figure 6(b). Thus, it is easy to see the trainees' adaptation to the training program. Moreover, this study found that the time for judging of participants was reduced significantly, and high exercise intensity is easier to let participants make mistakes than high task difficulty.

\section{Team Internal Contrast Test}

This study stipulated that the difference between the novices and the elite athlete is whether national effective rank has been obtained.

After lifting difficulty, as Figure 6(c), it is not difficult to find that the novices are less adaptable than the elite athletes, and male athletes are usually less adaptable than female athletes. In Figure 6(d), the elite athletes stable relatively, and the novices' level improves faster relatively. Critically, the differences between athletes arise when required proficiency has increased suddenly.

\section{Competition Contrast Test}

In 2016, five eighths of the team members had participated in this national competition. By contrast, only three eighths of the team members have participated in 2017. Therefore, it is relatively difficult to maintain the original results. And the results of the last two years' national competitions are shown in Table 1.

Compare the results of the two years, the team performance improved by nearly $13 \%$ and the proportion of the novices who gain the ranking is increased from $0 \%$ to $60 \%$. Moreover, the proficiency is positively related to the winning frequency.

Hence, the above training methods and the decomposition method are both effective. 


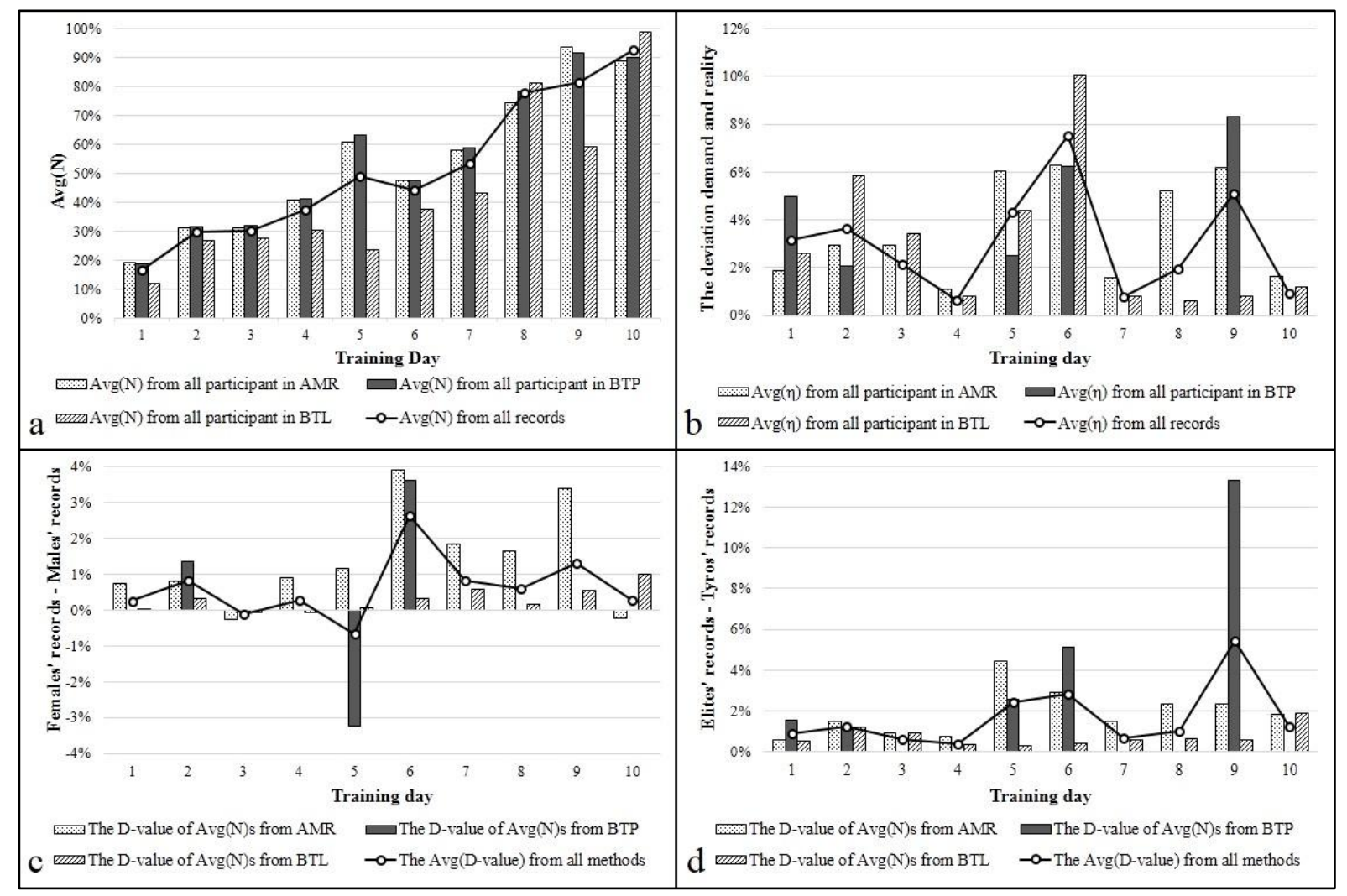

Figure 6 The result of STO from all participants

Table 1. The comparison of last two years' competitions results

\begin{tabular}{|c|c|c|c|c|c|c|c|c|}
\hline \multirow{2}{*}{$\begin{array}{l}\text { Member } \\
\text { ID }\end{array}$} & \multirow{2}{*}{$\begin{array}{l}\text { Take part in last } \\
\text { competition }\end{array}$} & \multirow{2}{*}{ Sex } & \multicolumn{6}{|c|}{ Competition Event } \\
\hline & & & $100 \mathrm{M}$ & Sprint & Middle & Point & Relay & Team \\
\hline $2016-1$ & $\sqrt{ }$ & M & $\times$ & $x$ & & $x$ & & $x$ \\
\hline 2016-2 & $\sqrt{ }$ & M & $11(5)$ & $x$ & $x$ & & $x$ & \\
\hline $2016-3$ & $x$ & M & $x$ & & & $x$ & & $x$ \\
\hline $2016-4$ & $x$ & M & & $x$ & $x$ & & $x$ & $x$ \\
\hline $2016-5$ & $\sqrt{ }$ & $\mathrm{F}$ & $x$ & $x$ & & $3(13)$ & & $x$ \\
\hline $2016-6$ & $\sqrt{ }$ & $\mathrm{F}$ & & $x$ & $9(7)$ & & $x$ & $x$ \\
\hline $2016-7$ & $\sqrt{ }$ & $\mathrm{F}$ & $x$ & $9(7)$ & & $9(7)$ & $x$ & \\
\hline $2016-8$ & $x$ & $\mathrm{~F}$ & $x$ & & $x$ & & & $x$ \\
\hline $2017-1$ & $\sqrt{ }$ & M & & $x$ & & $15(1)$ & $6(5)$ & $x$ \\
\hline $2017-2$ & $x$ & M & $x$ & $x$ & & $11(5)$ & & $x$ \\
\hline $2017-3$ & $x$ & M & $x$ & $x$ & $x$ & & $6(5)$ & \\
\hline $2017-4$ & $x$ & M & $x$ & & $x$ & & & $x$ \\
\hline $2017-5$ & $\sqrt{ }$ & F & $x$ & $x$ & & $5(11)$ & $6(5)$ & \\
\hline $2017-6$ & $\sqrt{ }$ & $\mathrm{F}$ & $x$ & $x$ & $10(6)$ & & & $x$ \\
\hline $2017-7$ & $x$ & $\mathrm{~F}$ & & $15(1)$ & $\times$ & & $6(5)$ & $x$ \\
\hline $2017-8$ & $x$ & $\mathrm{~F}$ & $x$ & & & $x$ & & $x$ \\
\hline
\end{tabular}

$* \times$ means athlete overtime, foul or after $15^{\text {th }}$ in the corresponding subject of the nation competition number in brackets means score (inverted scorecard, group items multiplied by 2 ) 


\section{Discussion}

This study has conducted three kinds of experiment to verify the effectiveness of using the three methods with the decomposition method. Currently, this study found that it is recommended that AMR method is added to the time reference. And there are also some problems such as insufficient samples and test time.

The above three training methods can be summed up as the special training tool for simple simulating the particular scene of the complex movement to effective quantitative training. This study deduces that other complex sports which is difficult to carry out can also be divided by decomposition method and be simulated special sub-skills to get effective training effect and reasonable evaluation index.

The instrument of the main experimental in this paper is STO. To facilitate the effective use and expansion of relevant practitioners, its source code and full design standards are placed on the public website ( $\underline{\text { GitHub}})$.

The further work will be divided into two parts. One is the establishment of the new simulation methods such as combination of route selection and Hamilton path. It is worth noting that Hamilton path is the most primitive model of orienteering. The other is the expansion of the methods such as the method which is shown in Figure 7. The complexity of the environment can be further simulated by increasing the impassable table and the passable benches.

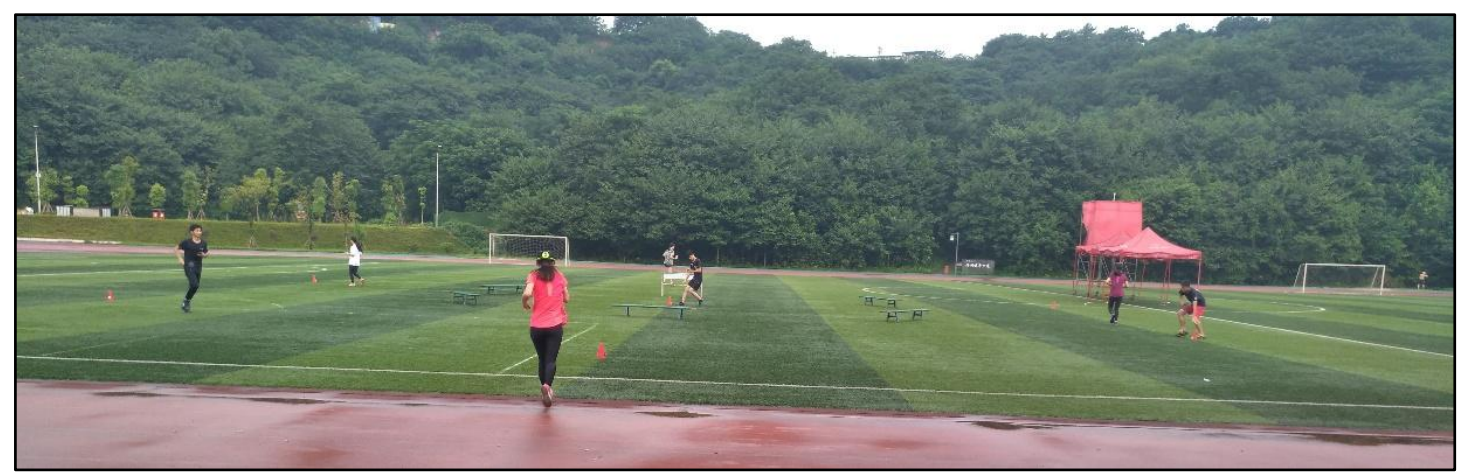

Figure 7. The expansion of BTP (BTP with obstacles) 


\section{Reference}

Asmaro, D., Mayall, J., \& Ferguson, S. (2013). Cognition at altitude: impairment in executive and memory processes under hypoxic conditions. Aviation, space, and environmental medicine, 84(11), 1159-1165.

Bengtsson, H., \& Atkinson, G. (1977). Orienteering for Sport and Pleasure.

Boes, R., Harung, H., Travis, F., \& Pensgaard, A. (2014). Mental and physical attributes defining world - class Norwegian athletes: Content analysis of interviews. Scandinavian journal of medicine \& science in sports, 24(2), 422-427.

Chi, Y.-Y., Lee, Y.-F., \& Tsai, S.-E. (2016). Study on High Accuracy Topographic Mapping via $U A V$-based Images. Paper presented at the IOP Conference Series: Earth and Environmental Science.

Cormen, T. H., Leiserson, C. E., Rivest, R. L., \& Stein, C. (2001). Introduction to algorithms second edition: The MIT Press.

Creagh, U., \& Reilly, T. (1997). Physiological and biomechanical aspects of orienteering. Sports Medicine, 24(6), 409-418.

Cych, P. (2006). Possibilities of and constraints on the application of GPS devices in controlling orienteering training. Proceedings of the National Academy of Sciences, 60(60), 1495-1502.

Dodge, M. (2017). Cartography in the Twentieth Century. The International Journal for the History of Cartography, 69(1), 114-118.

Fairbairn, D. (1990). Orienteering mapping and higher education. The Cartographic Journal, 27(2), 128-131.

Gilhooly, K. J., Wood, M., Kinnear, P. R., \& Green, C. (1988). Skill in map reading and memory for maps. The quarterly journal of experimental psychology, 40(1), 87-107.

Guzmán, J. F., Pablos, A. M., \& Pablos, C. (2008). Perceptual-cognitive skills and performance in orienteering. Perceptual and motor Skills, 107(1), 159-164.

Johnston, R. J., Watsford, M. L., Kelly, S. J., Pine, M. J., \& Spurrs, R. W. (2014). Validity and interunit reliability of $10 \mathrm{~Hz}$ and $15 \mathrm{~Hz}$ GPS units for assessing athlete movement demands. Journal of Strength \& Conditioning Research, 28(6), 1649-1655.

Komiyama, T., Sudo, M., Higaki, Y., Kiyonaga, A., Tanaka, H., \& Ando, S. (2015). Does moderate hypoxia alter working memory and executive function during prolonged exercise? Physiology \& behavior, 139, 290-296.

Krauß, T. (2015). Preprocessing of Satellite Data for Urban Object Extraction. ISPRS International Archives of the Photogrammetry, Remote Sensing and Spatial Information Sciences, XL-3/W2, 115-120.

Lauenstein, S., Wehrlin, J. P., \& Marti, B. (2013). Differences in horizontal vs. uphill running performance in male and female Swiss world-class orienteers. The Journal of Strength \& Conditioning Research, 27(11), 2952-2958.

Leger, L. A., Mercier, D., Gadoury, C., \& Lambert, J. (1988). The multistage 20 metre shuttle run test for aerobic fitness. Journal of sports sciences, 6(2), 93-101.

Macquet, A.-C., Eccles, D. W., \& Barraux, E. (2012). What makes an orienteer an expert? A case study of a highly elite orienteer's concerns in the course of competition. Journal of sports sciences, 30(1), 91-99.

Maling, D. H. (2016). Measurements from maps: principles and methods of cartometry: Butterworth-Heinemann.

McNeill, C. (2014). Orienteering: Skills-Techniques-Training: Crowood. 
Millet, G. Y., Divert, C., Banizette, M., \& Morin, J.-B. (2010). Changes in running pattern due to fatigue and cognitive load in orienteering. Journal of sports sciences, 28(2), 153-160.

Roelofs, E. J., Smith-Ryan, A. E., Melvin, M. N., Wingfield, H. L., Trexler, E. T., \& Walker, N. (2015). Muscle size, quality, and body composition: characteristics of division I crosscountry runners. Journal of strength and conditioning research/National Strength \& Conditioning Association, 29(2), 290.

Sension-Hall, D. (2011). Compass games: An introduction to orienteering skills. Journal of Physical Education, Recreation \& Dance, 82(1), 16-22.

Tønnessen, E., Svendsen, I. S., Rønnestad, B. R., Hisdal, J., Haugen, T. A., \& Seiler, S. (2015). The annual training periodization of 8 world champions in orienteering. International journal of sports physiology and performance, 10(1), 29-38.

Umek, A., \& Kos, A. (2016). The Role of High Performance Computing and Communication for Real-Time Biofeedback in Sport. Mathematical Problems in Engineering, 2016.

Wan, Z. (2014). O-Mopsi: Location-based Orienteering Mobile Game. M. Sc. thesis, University of Eastern Finland.

Zemková, E., Vilman, T., Kováciková, Z., \& Hamar, D. (2013). Reaction Time in the Agility Test Under Simulated Competitive and Noncompetitive Conditions. The Journal of Strength \& Conditioning Research, 27(12), 3445-3449.

Zentai, L. (2014). Implementation of cartographic and digital techniques in orienteering maps Cartography from Pole to Pole (pp. 19-29): Springer.

Zhang, H., Zhang, J., Huang, X., \& Liang, H. (2018). Infrastructure-less positioning in 3D space based on unpowered exoskeleton. Journal of Chongqing University of Technology(Natural Science), 32(3), 201-209. 